\title{
L’image récréative : des photos fantaisistes aux jeux virtuels
}

\section{Maria da Luz Correia*}

\begin{abstract}
Résumé
L'article qui suit est une tentative d'éclaircir l'idée d'image récréative, en l'exemplifiant à partir de la photo fantaisiste et des jeux vidéo. L'image récréative est corrélative aux transformations dans la notion d'image diagnostiquées par Walter Benjamin dans L'ouvre d'art à l'époque de sa reproduction mécanisée. La récréation visuelle est ici comprise comme une pratique qui, résultant de l'avènement des médias tels la photographie et le cinéma, procure la «distraction » et se sert du modèle du collage. Malgré leurs différentes positions dans la généalogie de l'image, la photographie fantaisiste des premières décennies du XXème siècle, qui nous occupera principalement ici, et les jeux virtuels, sont également exemplaires de cette notion.
\end{abstract}

Mots-clés :

jeu, simulacre, photographie, image numérique, montage

\begin{abstract}
:
This article aims to clarify the notion of re-creative image, by exemplifying it with fantasist photos and video games. The re-creative image corresponds to the transformations in the notion of image described by Walter Benjamin in L'oeuvre d'art à l'époque de sa reproduction mécanisée. The visual recreation is here understood as a practice that, resulting of the emergence of new media such as the photography, aims to "distract", and follows the model of collage. In spite of their different positions in the genealogy of image, fantasist photography of the first decades of the XXth century, which will be the main subject of this paper, and virtual games, are able to illustrate this notion.
\end{abstract}

\section{Keywords :}

play, simulation, photography, digital image, montage

Walter Benjamin ${ }^{1}$, dans les notes préparatoires à L'ouvre d'art à l'époque de sa reproduction mécanisée décompose l'art en deux versants : l' «apparence » et le «jeu». Selon le philosophe allemand, la perte de l'aura, conditionnée par le développement technique, se traduirait par un nouveau statut de l'image, basé sur un «flétrissement de l'apparence» et un gain pour «l'espace de jeu » : la distance transcendante de l'œuvre d'art est remplacée par une proximité ludique. Cette nouvelle image, corrélative à l'avènement des médias tels la photographie et le cinéma, implique une perception esthétique, qui n'est plus de l'ordre de la contemplation mais de l' « usage ». Elle correspond dans ses traits généraux à ce que nous entendons ici par image récréative.

Le terme de « récréation » est employé dans le domaine de l'expression visuelle pour la première fois pour faire référence à la photographie d'amateur. Ce sont des ouvrages de vulgarisation scientifique comme Les récréations photographiques d'Albert Bergeret, manuels qui enseignent au public non professionnel comment poser et truquer des portraits

\footnotetext{
* Chercheur au Centre d'Études de Communication et Société (CECS), Université du Minho, Braga, Portugal et au Centre d'Étude sur l'Actuel et le Quotidien (CEAQ) de l'Université Paris Descartes Sorbonne. mariadaluzcorreia@gmail.com

${ }^{1}$ Benjamin W., (1936) L'œuvre d'art à l'époque de sa reproduction mécanisée in Ecrits Français, Éditions Gallimard, Paris, 1991, pp. 242, 242.
} 
photographiques, et qui popularisent le terme ${ }^{2}$. Dans ces ouvrages, la notion de récréation est utilisée dans son acception la plus commune : emploi du temps libre, amusement, hobby... En effet, tout d'abord, l'idée de récréation met l'accent sur une image vouée à la pratique du jeu, en tant qu'action libre, éphémère et fictive, qui procure la distraction. Mais la notion d'image récréative renvoie aussi à un deuxième sens. Recréer c'est aussi créer à nouveau, « remettre en jeu » ${ }^{3}$, c'est-à-dire, s'approprier, bricoler, opérer avec des qualités secondes, « détourner » au sens de Guy Debord ${ }^{4}$.

Malgré leurs différentes positions dans la généalogie de l'image, la photographie fantaisiste du début du XXème siècle, qui nous occupera principalement ici, et les jeux virtuels, illustrent bien la double signification que l'on attribue à cette notion.

\section{La pellicule du rêve: portrait performé, portrait retouché}

Mais qu'entend-on par photographie fantaisiste ? On regroupe sous cette désignation tout un ensemble de procédés photographiques, pratiqués surtout pendant la première moitié du XXème siècle, qui se servaient des décors peints, miroirs, poses, objectifs et chambre noire pour insérer des visages familiers dans un monde onirique, mystérieux, exubérant, risible. Enfants bâtards du cinéma de Georges Méliès, leurs techniques combinent mise en scène et manipulation photographique. Diffusées dans les journaux illustrés et dans les cartes postales de l'époque, elles sont réalisées par les photographes amateurs, les studios et constituent aussi des attractions dans les fêtes foraines. Cristallisant le « fictionnel populaire » apprécié par Michel Maffesoli ${ }^{5}$, elles sont des « divertissements», des «trucs », des «passetemps», selon l'expression de C. Chaplot ${ }^{6}$.

Inscrites dans la tradition de l'amusement photographique, les photos fantaisie correspondent à un usage anthropologique de l'image, où le jeu est plus important que l'enregistrement du jeu. L'action ludique à l'œuvre dans les mises en scène exagérées ou dans

\footnotetext{
${ }^{2}$ Voir à ce propos Clément Chéroux, "Les récréations photographiques », Études photographiques, 5 | Novembre 1998, [En ligne], URL:http://etudesphotographiques.revues.org/index167.html. Consulté le 15 juillet 2010.

${ }^{3}$ Détourner selon Guy Debord équivaut précisément à « remettre en jeu ». G. Debord (1963). All the king's men. In DEBORD, Guy, Euvres, Gallimard, Paris, 2006, p. 617

${ }^{4}$ G. Debord (1956). Mode d'emploi du détournement. In DEBORD, Guy, Euvres, Gallimard, Paris, 2006, pp. 221-229.

${ }^{5}$ M. Maffesoli (1979), La conquête du présent. Pour une sociologie de la vie quotidienne. Desclée de Brower, Paris, 1998, p. 105.

${ }^{6}$ C. Chaplot, La photographie récréative et fantaisiste, Charles Mendel, Paris, 1904.
} 
l'exercice de retouche se donne souvent comme la première finalité de ces clichés. Ces photographies ont quelque chose du «savoir faire obstiné et insensé des cavaliers, des acrobates et des clowns », pour reprendre une expression de Adorno et Horkheimer ${ }^{7}$. Si la photographie est un «objet mélancolique », comme 1'appelle Susan Sontag ${ }^{8}$ - puisqu'elle s'oppose en quelque sorte au passage du temps - la photo récréative conjure bien tout «jeu triste $»^{9}$. Suivant la « loi de la répétition $»$ propre au ludique ${ }^{10}$, ces procédés sont répétitifs et bien souvent circonscrites à des occasions cycliques, comme les fêtes foraines. Les clichés mimés et retouchés mettent la technique de reproductibilité au service d'une improvisation libre, vacante, créative ou turbulente. Reprenant la classification de jeux de Roger Caillois ${ }^{11}$ jeux de compétition, jeux de hasard, jeux de simulacre et jeux de vertige - on peut reconnaître que la vocation ludique de ces portraits réside principalement dans le mimicry, le simulacre, l'illusion, cet exercice de faire semblant de. On verra encore qu'un tel exercice engendre souvent le vertige ce que Roger Caillois appelle l'ilinx: c'est le «déséquilibre », le «tremblement des corps »dans les mots de Moisés de Lemos Martins ${ }^{12}$.

On se propose de distinguer deux différents types de photos à l'intérieur de la désignation générale de photographie fantaisiste. D'un côté, le portrait performé ${ }^{13}$ qui repose sur la mise en scène, et, d'un autre côté, la photographie retouchée qui utilise les effets optiques et chimiques.

Le portrait performé est dépendant du déguisement, du décor, de la mimique... Il est réalisé souvent au milieu du bric-à-brac des studios photographiques qui dans les premières années du XXème siècle se remplissent de « colonnes », de «palmiers », et font poser leurs clients dans des faux jardins d'hiver - ceci nous est décrit notamment par Walter Benjamin

\footnotetext{
${ }^{7}$ M. Horkheimer et T. Adorno (1944), La dialectique de la raison, Éditions Gallimard, Paris, 1974, p.152.

${ }^{8}$ Sontag S., Sur la photographie, Christian Bourgois Éditeur, Paris, 2008, p.77.

9 À ce propos, voir la notion de « jeu triste » (trauerspiel) présentée par Walter Benjamin in Origine du drame baroque allemand (1925), Champs-Flammarion, Paris, 1985.

${ }^{10} \mathrm{Cf}$. W. Benjamin O brinquedo e o jogo, Notas à margem de uma obra monumental in Sobre arte, técnica, linguagem e política. Relógio d'Água, Lisboa, 1992.

11 R. Caillois, Les jeux et les hommes. Le masque et le vertige, Gallimard, Paris, 1958, pp. 25 - 66.

12 M. Lemos Martins «Declinar a vibração da vida»., in A. Gonçalves. Vertigens. Para uma sociologia da perversidade, Grácio Editor, Coimbra, 2009, p. 7.

13 On reprend ici une désignation de Clément Chéroux, appliquée à la photographie des surréalistes, qui s'amusaient à se déguiser, à faire des poses excentriques, ou à participer aux jeux forains: "Les portraits de cette dernière catégorie rappellent d'ailleurs, par bien des aspects, certaines photographies de performances, d'action ou de happenings des années 1960 à 1980. Ce registre de représentation collective, que les dadaïstes avaient occasionnellement expérimenté et que les surréalistes ont beaucoup plus systématiquement exploité, offre en fait les premiers exemples de portraits performés de l'art du XXème siècle." C. Chèroux, « L'action collective, La photographie par tous, non par un », in Q.Bajac, C. Chèroux, G. Le Gall, M. Poivert, La Subversion des images. Surréalisme, photographie, film, Éditions du Centre Pompidou, Paris, 2009, p. 27.
} 
dans Petite Histoire de la Photographie ${ }^{14}$. À partir des années 20, ces portraits sont aussi souvent pratiqués dans les fêtes foraines et parcs d'attractions. Les «passe-têtes ${ }^{15}$, des décors peints percés de trous, qui simulaient des plages, des voitures, des avions, et auxquels le visage des personnes était juxtaposé, sont une des modalités du portrait forain.

Il reste encore les photographies prises dans les labyrinthes de miroirs déformants et celles résultant du tir photographique. Ce dernier était une attraction populaire dans les fêtes foraines qui se présentait comme un stand de tir commun mais dont la cible, lorsqu'elle était touchée par le plomb, actionnait un système photographique qui fixait instantanément le tireur en un éclair. Le pendant ludique du portrait performé, qui était souvent pratiqué en groupe, se retrouve dans l'exercice du faire comme si : comme si l'on conduisait une voiture, ou même dans le cas du tir photographique, comme si l'on était une star des westerns de l'époque. Comme le mentionne Pierre Mac Orlan, convoqué par Clément Chéroux ${ }^{16}$, les baraques des jeux photographiques «sont des temples de 'vies imaginaires'». Mais il est vrai que l'on se trouve aussi dans un terrain de socialisation du vertige, selon l'idée de Roger Caillois ${ }^{17}$. Par exemple, revenons au tir photographique. Au-delà du jeu d'adresse et de l'expérience physique de tirer, la réversibilité des positions de ce jeu est déconcertante. Entre le tireur qui vise l'appareil photographique et l'appareil qui tire le portrait s'ouvre une sorte de mise en abyme, qui déroute. Clément Chéroux (2009 : 7) dans son livre La Photo qui fait mouche, évoque, non sans raison, un «vertige de l'auto-destruction ». Dans ces portraits, l'éclat du flash répond comme par effet de ricochet au tir de la carabine, le modèle se confond avec le photographe, le tireur devient sa propre cible.

Quant à la photographie manipulée dans la chambre noire, qui a commencé par être un passe-temps des photographes amateurs, elle exposait la figure humaine et les objets en général aux effets optiques et chimiques. Si la photographie est en soi une «manifestation de la force du double» (pour reprendre la définition de Michel Maffesoli ${ }^{18}$ ) les photographies fantaisistes en font la preuve, démultipliant un même visage dans une seule photographie. Le coloriage des épreuves en noir et blanc donne un éclat bizarrement technicolor aux corps, qui

\footnotetext{
${ }_{15}^{14}$ W.Benjamin, Petite histoire de la photographie, Paris, Société Française de la Photographie, 1996, pp. 17, 18.

${ }^{15}$ Cette désignation nous est signalée par Clément Chéroux. L'auteur ajoute une note à propos de cette appellation, qu'on reproduit ici: «Je remercie François Cheval, le conservateur du musée Nicephore-Niépce de Chalon-sur-Saône d'avoir attiré mon attention sur cette appellation. L'équivalent anglais Poke your head était également employé par les photographes forains outre-Manche. » . C. Chèroux, " Portraits en pied... de nez ", Études Photographiques, $16 \quad \mid$ Mai 2005, [En ligne], http://etudesphotographiques.revues.org/index721.html. Consulté le 09 juillet 2010.

${ }^{16}$ C.Chèroux, op. cit.

${ }^{17}$ R.Caillois, op. cit., pp. 73, 210.

${ }^{18}$ M. Maffesoli, op. cit.p.p.104.
} 
ressemblent ainsi à des poupées, à des automates. Par l'effacement partiel de l'épreuve, on insérait aussi des spectres à la photographie - la photographie spirite s'est développée sur ce principe. La déformation des corps, qui se faisait par exemple en utilisant les « objectifs anamorphotes $»^{19}$, était aussi un truquage fréquent. Finalement, les tirages combinés, les doubles impressions et l'utilisation de papier sensible illustré permettaient de refaire des êtres disproportionnés, hybridés, risibles: un animal inconnu, des femmes avec des mains gigantesques, des hommes à trois jambes. Au-delà du jeu d'illusion de ces cadavres exquis, le vertige apparaît lié à cette surprise de se voir porter un corps étranger, souvent inhumain, à l'inquiétude de se découvrir à côté de soi-même, ou encore, au «plaisir ambigu du frisson et du mystère », selon l'expression de Roger Caillois ${ }^{20}$. Ces portraits démontés et remontés aboutissent à une dérision de la figure humaine et matérialisent un corps qui, au rythme des assauts chimiques et optiques, reconstruit « la monstruosité qui nous habite », pour reprendre les mots de Moisés de Lemos Martins ${ }^{21}$. Finalement, inspirant la photographie de certains surréalistes comme Raoul Ubac, Man Ray ou Hans Bellmer, les photographies fantaisistes manipulées se prêteraient encore à une confrontation aux commentaires de Rosalind Krauss et de Georges Didi-Huberman à propos l'exploration photographique de l'«informe» ${ }^{22}$.

\section{Photographies et jeux virtuels : du devant au dedans}

Les photographies fantaisistes enregistrent une réunion du réel et de l'imaginaire, et elles donnent aussi à voir l'image de la rencontre entre l'homme et la machine, que toute photographie elle-même suppose ${ }^{23}$. Le trompe l'oeil à l'œuvre dans les clichés mimés et truqués, apparus peu après l'avènement de l'appareil photographique, s'appuie, en effet, sur la

19 C. Chaplot, op. cit., p. 81.

20 R. Caillois, op.cit., p. 205.

21 M. Lemos Martins, “Ce que peuvent les images. Trajet de l'un au multiple”, in Les Cahiers Européens de 1'Imaginaire, nº1, CNRS, Paris, 2009, p. 162.

${ }^{22}$ L' « informe » est une notion introduite par Georges Bataille qui, d'après les lectures de Krauss et DidiHuberman renverrait à un réel non humanisé, et qui mettrait en évidence les «pouvoirs du lieu » comme le dit Didi-Huberman - hétérogène, inconnu, disproportionnel - par opposition à la fragilité de l'humain. L'informe serait ainsi le réel non-maîtrisable, non définissable, insubordonné, un espace qui défigure et reconfigure en permanence.

R. Krauss, «Corpus Delicti» in Krauss R., Explosante-Fixe, Photographie et Surréalisme, Centre Georges Pompidou/Hazan : 55 - 112, Paris : 1985.

G. Didi-Huberman, La ressemblance informe, ou le gai savoir selon Georges Bataille. Macula, Paris, 1995.

${ }^{23}$ Il est opportun de rappeler les notes de Benjamin à propos de Charlie Chaplin : selon Benjamin, les gestes saccadés de Chaplin ne pouvaient apparaître qu'à l'intérieur de la structure technique du cinéma - le montage. Or, de façon semblable, les créatures hybrides qui figurent dans les portraits fantaisistes du début du siècle ne pouvaient survenir que dans le contexte de la technique et, très particulièrement, de la stratégie du photomontage. W. Benjamin, (1936) L'euvre d'art à l'époque de sa reproduction mécanisée in Ecrits Français, Éditions Gallimard : 149 - 248, Paris, 1991, pp. 225, 226. 
nature spécifique de la photo. Rosalind $\mathrm{Krauss}^{24}$ explicite que sur «l'arbre généalogique des images », la photographie se situe du côté des «empreintes de main, des masques mortuaires, du Suaire de Turin». Or, cet aspect indiciel de la photographie donne à l'image un effet de réalité, et lui fait perdre en même temps la distance symbolique qui la caractérisait: l'image n'est plus la représentation de la chose mais la trace de la chose. Les photos fantaisie utilisent les décors peints et le photomontage, mais sauvegardent la continuité de la surface de l'épreuve finale, donnant l'impression que l'image « demeure une information sur la réalité ». ${ }^{25}$ En croisant l'autorité du cliché avec la liberté du truquage, les photos fantaisie jouent à faire preuve du surréel, à réaliser l'irréel, à actualiser le virtuel.

La rencontre entre l'homme et la machine se déroule-t-elle dans l'image numérique de la même manière que dans ces clichés analogiques? Dans une exposition intitulée Dreamlands qui s'est déroulée entre mai et août 2010 au Centre Pompidou, à Paris, on retrouvait des images des amusements photographiques des fêtes foraines présentées à quelques mètres des projections du monde virtuel Second Life $^{26}$. Certes, il y a des aspects en commun entre ces deux images techniques. S'emparant à nouveau de l'image en tant qu'entité ludique, les jeux vidéos et les mondes en réseau tel Second Life de Linden Lab mettent également les technologies de l'image au service des cristaux du faux ${ }^{27}$, de ce jeu du faire comme si, propre aux enfants et aux sociétés dites primitives. Les écrans en 3D de Second Life, comme les clichés d'autrefois, miment souvent les moments les plus anodins de la vie, comme conduire une voiture. Libérées de leur fonction de représentation, les épreuves manipulées du début du siècle et les jeux vidéo comme World of WarCraft imaginent et conjurent des monstres. Cependant, en remplaçant la magie de l'enregistrement par l'hallucination du direct, les jeux virtuels accroissent les risques - de possession, d'aliénation - suscités par la « coalition toute-puissante du simulacre et du vertige $»^{28}$.

\footnotetext{
${ }^{24}$ R. Krauss, «La photographie au service du surréalisme» in Krauss R.. Explosante-Fixe, Photographie et Surréalisme. Centre Georges Pompidou/Hazan, Paris, 1985, p. 31.

${ }^{25}$ Ibidem, p. 28.

${ }^{26}$ Cette exposition, qui s'est déroulée entre le 5 mai 2010 et le 9 août 2010, présentait dans la même salle, entre autres, des diapositives reproduisant des photographies des surréalistes dans les passe-têtes des fêtes foraines et des images de RMB City, une communauté artistique sur Second Life créée par Cao Fei.

${ }^{27}$ À ce propos, voir G. Deleuze «Les puissances du faux » in Cinéma 2, L'image-temps. Les éditions de Minuit, Paris, 1985.

${ }^{28}$ Roger Caillois, op. cit., p. 224.

Ce constat confirme, d'ailleurs, la « synergie entre archaïsme et développement technologique » mentionnée par Michel Maffesoli.

M. Maffesoli L'instant éternel. Le retour du tragique dans les sociétés postmodernes. Denoël, Paris, 2000, p.187.
} 
La distance entre le simulacre opéré par les photographies fantaisistes et celui produit par les jeux virtuels correspond à la différence remarquée par certains auteurs entre la société du spectacle de Guy Debord et la société hyper réelle de Jean Baudrillard ${ }^{29}$ : pour Regis Debray $^{30}$ notamment, dans la première, on est encore « devant l'image », dans la deuxième, on est «dans le visuel ». Le jeu photographique se servait de la preuve magique de l'enregistrement pour faire croire et donner un impact de « choc » (Benjamin,1992) à un réel créé : un tel effet se restreignait, cependant, à une extériorité artificielle et différée. La sensation de soudaine étrangeté qui envahit celui qui regarde son portrait déformé ou la joie vide de celui qui comble les trous du panneau peint ne provoque pas un dédoublement de la conscience, ni une personnalité multiple. Par contre, le joueur de Second Life qui choisit de beaux organes pour son avatar est effectivement à l'intérieur des masques instables qu'il porte, faisant preuve de cette «nouvelle profondeur de soi », selon les mots de Derrick de Kerckove (2000: 88). L'impression d'étourdissement du visiteur des attractions photographiques foraines ne réside pas souvent dans l'image mais plutôt dans le hors-dechamp de l'image; or, dans les jeux vidéo l'image occupe tout le champ. Par exemple, le joueur du tir photographique tenait la carabine, la mettait en joue, prenait sa mire, tirait, écoutait le bruit du tir, se faisait éblouir par l'éclat du flash... Celui qui joue avec des pistolets lasers ou optiques sur les consoles Playsation ou sur les bornes d'arcades, tient dans la main le pistolet et écoute aussi le bruit du tir. Mais cette interaction ne se fait pas sans l'échange permanent et simultané avec l'écran, sorte de l'autre côté du miroir qu'il habite lorsqu'il commence à jouer. Finalement, dans la plupart des modalités des jeux photographiques, même si le décor ou l'épreuve manipulée peuvent servir de surface de projection, l'exercice du faire semblant de est celui d'un jeu bouffon, selon l'expression employée par Roger Caillois (1958: 208) à la suite de G. Buraud. Les masques photographiques prétendent davantage faire rire Sancho Pança que convaincre ou ensorceler Don Quichotte. Or, les jeux vidéo ne se contentent pas de la mascarade maladroite des photographies récréatives. Dans les jeux virtuels, l'extériorité qui ment et qui fausse fait place à une intériorité qui enveloppe et immerge ; les «fantasmes du réalisme ${ }^{31}$ sont conjurés par le réalisme du fantasme ; la fétichisation du réel est remplacée par la production du réel ; et, enfin, d'une reproduction différée on passe à une présence simultanée.

\footnotetext{
${ }^{29}$ G. Debord, La Société du Spectacle (1967) in G. Debord Euvres, Gallimard, Paris, 2006 : 765 - 859 ; J. Baudrillard, Simulacres et Simulation, Galilée, Paris, 1981.

${ }^{30}$ R. Débray, Vie et mort de l'image: Une histoire du regard en Occident, Gallimard, Paris, 1992, p. 383.

${ }^{31}$ L'expression est utilisée par Jean-François Lyotard, à propos de la photographie et du cinéma: « Ainsi se multiplient les effets de la réalité ou si l'on préfère les fantasmes du réalisme ». J-F. Lyotard, Le postmoderne expliqué aux enfants, Paris, Galilée, 2005, p.19.
} 


\section{Monter des images : le réel rejoué et rejouable}

Malgré leurs différences, photographies fantaisistes et jeux virtuels sont les deux pratiques qui, un peu comme les situations et les jeux débordiens, mettent les techniques de reproductibilité au service d'une diminution des moments ennuyeux ou même à une exploration d'émotions nouvelles ${ }^{32}$. Fictions visuelles, les unes comme les autres sont des images récréatives, tout d'abord en raison de leur jeu de simulacre : leur qualité ludique leur permet de perméabiliser la réalité, bien qu'à différents degrés. Or, cette déstabilisation du réel ne serait pas accomplie sans une présentation du monde qui suive les techniques du découpage, du collage, du montage, de l'appropriation, du détournement. C'est par le biais de ces actions que le réel devient jeu, que la réalité (decontextualisée, recontextualisée) s'hybride de fantaisie, que le familier (découpé, collé) apparaît comme étrange... Ainsi, jeux virtuels et photographies fantaisistes sont des images récréatives, non seulement parce qu'elles jouent à faire semblant de, mais aussi parce qu'elles remettent en jeu, se dédiant à cet autre exercice du il était une fois, encore une fois.

Mettant en image des êtres de la mythologie populaire et tissant des liens avec le récit traditionnel, le conte et la légende ${ }^{33}$, photographies fantaisistes et jeux virtuels sont des images sans auteur, dont le code esthétique suit le modèle de la "performance » plutôt que celui de l' « authorship » ${ }^{34}$. Comme dans le cinéma, la nature illusionniste des photographies fantaisistes et des jeux numériques - « une nature au second degré », comme l'appelle Walter Benjamin $^{35}$ - est le résultat du «découpage » : c' est le moyen de présenter une réalité artificielle. Les représentations photographiques et numériques participent comme le dirait

\footnotetext{
${ }^{32}$ Dans le Rapport sur la Construction des Situations, Guy Debord semble attribuer un sens identique à l'idée de «jeu » et à la notion de "situation»: les deux sont éphémères et se destinent à produire des nouveaux sentiments et des passions inédites. L'auteur explicite que pour que les situations produisent des « sentiments inexistants auparavant », il faut qu'il y ait une « nouvelle application des techniques de reproduction ».

G. Debord (1957). Rapport sur la construction des situations et sur les conditions de l'organisation et de l'action de la tendance situationniste internationale. In G.DEBORD, Euvres, Gallimard, Paris, 2006, pp. 322328.

${ }^{33}$ À ce propos voir : P. Mpondo-Dicka, «Les scènes cinématiques dans les jeux vidéo, Analyse sémiotique de quelques formes et fonctions » in S. Genvo, Le game design de jeux vidéo, Approches de l'expression vidéo ludique, L'Harmattan, Paris, 2005, pp. 209- 235.

${ }^{34}$ C'est Giorgio Agamben qui nous rappelle cette dualité : selon l'auteur, la dichotomie performance/authorship est introduite par l'école américaine "qui - sur les traces de Milman Parry et de Marcel Jousse - a apporté une contribution si originale à l'étude de la poésie orale." G. Agamben, Image et mémoire. Écrits sur l'image, la danse et le cinéma, Desclée de Brouwer, Paris, 2004, p.76.

${ }^{35} \mathrm{~W}$. Benjamin, (1936) L'œuvre d'art à l'époque de sa reproduction mécanisée in Ecrits Français, Éditions Gallimard, Paris, 1991, pp. 204, 205.
} 
Levy-Strauss à la transposition de la pensée «bricoleuse» sur le terrain visuel. ${ }^{36}$ Elles opèrent avec des propriétés secondes, s'accordant avec la notion débordienne de « détournement », c'est-à-dire, l'appropriation et la réorganisation d'éléments déjà existants. Les images à jouer des jeux virtuels et des clichés fantaisie sont aussi des images à monter, à reconstituer : en juxtaposant notre tête à un décor peint, en défigurant un portrait dans la chambre noire, en agissant sur un environnement visuel en 3D, lui-même constitué par un amalgame de récupérations visuelles.... Par le biais du photomontage et du montage interactif, on désorganise et réorganise le réel, démonte et remonte le tissu du monde, rassemble ce qui ne se ressemble pas, fragmente et multiplie, défigure et transfigure.

Les photos fantaisistes s'appuient sur la technique photographique et très particulièrement, comme on a déjà noté, sur la continuité de l'épreuve photographique, pour assembler dans un même portrait des rebuts de corps disparates, des fragments de scènes diverses. Si le montage cinématographique consiste, comme le mentionne Walter Benjamin en des «images discontinues» qui «s'enchaînent au sein d'une succession continue»" ${ }^{37}$, dans le photomontage et dans les photos jouées et posées à l'aide de décors, on retrouve des morceaux d'images coupés et interrompus qui s'assemblent dans la surface ininterrompue de l'épreuve photographique. Il s'agit d'un patchwork sans coutures, d'un assemblage de citations sans guillemets, d'un collage dont auraient disparu les marques de colle et de ciseaux. C'est « une image » sans « cicatrices», selon la juste métaphore de Philipe Alain Michaud $^{38}$. En décomposant et recomposant une image en des agencements irréels (par des procédés qui sont souvent répétitifs et formulaires), la photographie fantaisiste réussit à acquérir une valeur de réalité, à provoquer des émotions - l'effet de « choc » que Benjamin attribue aux images techniques ne désigne que l'intensité de ces émotions.

Les jeux vidéo se servent du montage interactif, lui-même dépendant de périphériques, contrôleurs et interfaces qui permettent au joueur d'agir sur l'environnement visuel, sonore. Les images et les sons sont à nouveau divisés et recomposés dans une succession, mais cette succession est désormais elle-même interrompue, démultipliée, passible d'être expérimentée en plusieurs sens, par son utilisateur. Il s'agit d'un puzzle dont le plan se rétablit constamment, un jeu de marionnettes dont les gestes sont en partie programmés, en partie

\footnotetext{
${ }^{36}$ «La vogue intermittente des collages nés au moment où l'artisanat expirait pourrait n'être de son côté, qu'une transposition du bricolage sur le terrain des fins contemplatives »(C. Levy-Strauss La pensée sauvage. Plon, Paris, 1962, p. 44)

${ }^{37}$ W. Benjamin, op. cit., p. 225.

38 P.A. Michaud, «Table de Montage, La coalescence et la suture. » in Bajac Q., Chéroux C., Le Gall G., Poivert M., La Subversion des images. Surréalisme, photographie, film. Éditions du Centre Pompidou, Paris, 2009, p. 174
} 
improvisés. L'apparence des personnages, la séquence d'actions et de mouvements, les graphismes... - tout ça est à la fois reproduction de la réalité, création du game-design du jeu, réaction, présence et appropriation du joueur. L'effet réaliste des compositions photographiques fantastiques est ici remplacé par l'immersion des mondes fictionnels interactivement engendrés.

Les affabulations photographiques inspirèrent les dadaïstes et les surréalistes. Les jeux vidéos sont aujourd'hui des nouveaux modèles narratifs pour un artiste comme Bill Viola, qui travaille depuis 5 ans sur un jeu vidéo, The Night Journey, ou pour un réalisateur comme Chris Marker, qui habite à Second Life. Malgré leurs aspects stéréotypés et prévisibles l'utilisation des «passe-têtes» ou du papier illustré dans le photomontage aboutit invariablement aux mêmes résultats, et les jeux vidéo correspondent bien souvent à des intrigues pauvres - la présence du modèle du collage introduit dans ces images une poursuite de l'aventure et une exploration de la surprise qui n'est pas à négliger. En fait, le montage repose sur la possibilité positive et optimiste de rejouer, s'accordant avec une compréhension de la vie en tant que force de transformation. Même si jeux numériques et photos fantaisistes correspondent à différents moments dans l'histoire de l'image - celui de la colle et des ciseaux et celui du copy and paste - tous deux s'accordent à une notion de l'image vouée à des « viveurs $»^{39}$ : des viveurs d'un réel qui ne trouve désormais d'autre définition que dans la virtualité, le désir, le possible...

\footnotetext{
${ }^{39}$ Viveurs est le terme employé par Guy Debord dans le Rapport sur la Construction des Situations: "La situation est ainsi faite pour être vécue par ses constructeurs. Le rôle du public, sinon passif du moins seulement figurant, doit y diminuer toujours, tandis qu'augmentera la part de ceux qui ne peuvent être appelés des acteurs mais dans un sens nouveau de ce terme, des viveurs."En même temps, Lifers est le terme aussi utilisé pour désigner les habitants de Second Life - cette désignation nous est notamment rappelée par Cayeux.

G. Debord, op. cit., p. 326.

Agnès Cayeux \& C. Guibert, Second Life, Un monde possible, Les petits matins, Paris, 2007.
} 


\section{Bibliographie}

Agamben G., Image et mémoire. Écrits sur l'image, la danse et le cinéma, Desclée de Brouwer, Paris, 2004.

Benjamin W., (1936) L'euvre d'art à l'époque de sa reproduction mécanisée in Ecrits Français, Éditions Gallimard : 149 - 248, Paris, 1991.

Benjamin W., Petite histoire de la photographie, Paris, Société Française de la Photographie, 1996.

Caillois R., Les jeux et les hommes. Le masque et le vertige, Gallimard, Paris, 1958.

Cayeux d'Agnès de \& Guibert C., Second Life, Un monde possible, Les petits matins, Paris, 2007.

Chaplot C., La photographie récréative et fantaisiste, Charles Mendel, Paris, 1904.

Chèroux C., «Portraits en pied... de nez », Études Photographiques, 16 | Mai 2005, [En ligne], URL : http://etudesphotographiques.revues.org/index721.html. Consulté le 09 juillet 2010.

Chèroux C., La photographie qui fait mouche, Carnets de Rhinoceros, Paris, 2009.

Chèroux C., "L'action collective, La photographie par tous, non par un », in Bajac Q., Chèroux C., Le Gall G., Poivert M., La Subversion des images. Surréalisme, photographie, film, Éditions du Centre Pompidou, Paris, 2009.

Debord G. (1956). Mode d'emploi du détournement. In DEBORD, Guy, Euvres, Gallimard : 221 - 229, Paris, 2006.

Debord G. (1957). Rapport sur la construction des situations et sur les conditions de l'organisation et de l'action de la tendance situationniste internationale. In DEBORD G., Euvres, Gallimard : 309-328, Paris, 2006.

Débray, R. Vie et mort de l'image: Une histoire du regard en Occident. Gallimard, Paris, 1992.

De Kerckhove D., L'intelligence des réseaux, Odile Jacob, Paris, 2006.

Didi-Huberman, G.. La ressemblance informe, ou le gai savoir selon Georges Bataille. Macula, Paris, 1995.

Horkheimer M. \& Adorno T. (1944), La dialectique de la raison, Éditions Gallimard, Paris, 1974.

Krauss R., "La photographie au service du surréalisme" in Krauss R.. Explosante-Fixe, Photographie et Surréalisme. Centre Georges Pompidou/Hazan :13-54, Paris, 1985.

Krauss R., "Corpus Delicti” in Krauss R., Explosante-Fixe, Photographie et Surréalisme, Centre Georges Pompidou/Hazan : 55 - 112, Paris : 1985. 
Levy-Strauss C. La pensée sauvage. Plon, Paris, 1962.

Maffesoli M. L'instant éternel. Le retour du tragique dans les sociétés postmodernes. Denoël, Paris, 2000.

Maffesoli M. (1979), La conquête du présent. Pour une sociologie de la vie quotidienne. Desclée de Brower, Paris, 1998.

Martins, M. L., "Ce que peuvent les images. Trajet de l'un au multiple”, in Les Cahiers Européens de l'Imaginaire, $\mathrm{n}^{\circ} 1$, CNRS, Paris, pp. 158-162, 2009.

Martins M. "Declinar a vibração da vida.", in GONÇALVES, Albertino. Vertigens. Para uma sociologia da perversidade, Grácio Editor, Coimbra, 2009.

Michaud P.A., "Table de Montage, La coalescence et la suture." in Bajac Q., Chéroux C., Le Gall G., Poivert M., La Subversion des images. Surréalisme, photographie, film. Éditions du Centre Pompidou, Paris, 2009.

Sontag S., Sur la photographie, Christian Bourgois Éditeur, Paris, 2008. 